\title{
Araneofauna de pomares de laranja Valência nos Vales do Caí e Taquari, Rio Grande do Sul, Brasil
}

\author{
Ana P. Ott ${ }^{1}$, Ricardo Ott ${ }^{2} \&$ Vera R. S. Wolff ${ }^{3}$
}

1. Departamento de Fitossanidade, Faculdade de Agronomia, Universidade Federal do Rio Grande do Sul, Av. Bento Gonçalves 7712 , Prédio 41201, 91540-000 Porto Alegre, RS. (ana.ott@ufrgs.br)

2. Museu de Ciências Naturais, Fundação Zoobotânica do Rio Grande do Sul, Rua Dr. Salvador França 1427, 90690-000 Porto Alegre, RS. (rott@fzb.rs.gov.br)

3. Laboratório de Entomologia, Fundação Estadual de Pesquisa Agropecuária (Fepagro), Rua Gonçalves Dias 570, $90130-060$ Porto Alegre, RS. (vera-wolff@ fepagro.rs.gov.br)

\begin{abstract}
Araneofauna of Valência orange orchards at the Caí and Taquari Valleys, Rio Grande do Sul, Brazil. Assemblages of spiders of the herbaceous vegetation were sampled by sweeping method in two sweet orange orchards (Citrus sinensis) in the south of Brazil. Each sampled orchard is characterized by receiving a different handling type: "traditional" or "ecological". In addition it was sampled the assemblage of spiders of the orange trees of the orchard with the "ecological" handling with the beating method. A total of 3,876 spiders were collected, 2,379 in the orange trees and 1,497 in the herbaceous vegetation; 17 families and 99 species were registered; Oxyopes salticus Hentz, 1845 (Oxyopidae) was the most abundant spider in herbaceous vegetation and Sphecozone cristata Millidge, 1991 (Linyphiidae) the most abundant in the orange trees. Cursorial spiders were the most abundant guild at both herbaceous vegetation and orange trees. The araneofauna of herbaceous vegetation in the different handling orchards did not present significant differences in diversity ( $H^{\prime}=2.13$ - "ecological"; 2.24 - "traditional"); the diversity was smaller in the orange trees $\left(H^{\prime}=1.95\right)$. Perhaps because of different sampling methods at different microhabitats, the Jaccard's Index indicates low similarity between assemblages $(17.5 \%)$.
\end{abstract}

KEYWORDS. Araneae, Citrus sinensis, diversity, herbaceous vegetation, agroecology.

RESUMO. Assembléias de aranhas da vegetação herbácea foram amostradas com rede-de-varredura em dois pomares de laranja doce (Citrus sinensis) no sul do Brasil. Cada pomar amostrado é caracterizado por receber um diferente tipo de manejo: "tradicional" ou "ecológico". Adicionalmente, foi amostrada a assembléia de aranhas das laranjeiras do pomar com manejo "ecológico" através do uso de guarda-chuva japonês. No total foram coletadas 3.876 aranhas, 2.379 nas laranjeiras do pomar de manejo "ecológico" e 1.497 junto à vegetação herbácea de ambos pomares; foram registradas 99 espécies de 17 famílias de aranhas; Oxyopes salticus Hentz, 1845 (Oxyopidae) foi a aranha mais abundante na vegetação herbácea e Sphecozone cristata Millidge, 1991 (Linyphiidae) a mais abundante nas laranjeiras. Aranhas errantes foram mais abundantes em ambos tipos de vegetação. A araneofauna da vegetação herbácea nos pomares com diferentes manejos não apresentou diferenças significativas na diversidade ( $\mathrm{H}^{\prime}=2,13$ - "ecológico"; 2,24 - "tradicional"); a diversidade foi menor nas laranjeiras $\left(H^{\prime}=1,95\right)$. Em razão de terem sido utilizados diferentes métodos de coleta nas amostragens entre os microhabitats, o índice de Jaccard $(17,5 \%)$ indicou baixa similaridade entre as assembléias.

PALAVRAS-CHAVE. Araneae, Citrus sinensis, diversidade, vegetação herbácea, agroecologia.

A simplificação extrema dos sistemas agrícolas caracterizados por monoculturas requer constante intervenção humana e grande quantidade de insumos agrícolas para manutenção de sua estabilidade e produtividade. Os efeitos indesejáveis sobre o meio ambiente e os altos custos de manejo têm minado a sustentabilidade das práticas agrícolas, sendo que a solução mais imediata para este problema parece estar no uso de métodos de produção orgânicos ou "ecológicos" (Altieri et al., 2003; GLIESSMANN, 2000). À medida em que estes métodos vem sendo valorizados, cresce a atenção dispensada à riqueza de espécies encontrada nos sistemas agrícolas. O estudo da diversidade destes ecossistemas tem cada vez mais despertado o interesse dos pesquisadores, que sugerem que o incremento da riqueza de espécies possa ser uma das chaves para o manejo integrado de pragas (ALTIERI et al., 2003).

As aranhas são um dos principais grupos da fauna de artrópodes dos sistemas agrícolas e, como predadores, podem alimentar-se de 40 a $50 \%$ da biomassa disponível de insetos, representando um papel importante no controle biológico de espécies causadoras de danos às culturas de importância econômica (RIECHERT \& LOCKLEY, 1984; Tarabaev \& Sheykin, 1990; Green, 1996; VANGSGAARD, 1986).

O grande número de adaptações das aranhas a diferentes microhabitats e ambientes com complexidade arquitetural variada podem contribuir decisivamente para a elevada abundância e riqueza de espécies deste grupo, mesmo em sistemas agrícolas monoculturais (TURNBULL, 1973; RyPSTRA et al., 1999). Estudos da araneofauna em sistemas agrícolas corroboram a afirmativa acima, registrando uma alta riqueza de espécies destes organismos em agrossistemas (GREEN, 1996; RinALDI \& FORTI, 1997; RINALDI et al., 2002; RINALDI \& RUIZ, 2002).

Aranhas têm sido estudadas como agentes de controle biológico de pragas (Hanna et al., 2003; Sunderland \& SAmu, 2000); destaca-se o sucesso de estudos experimentais na América do Norte com a predação de aranhas do gênero Theridion (UC DAVIS, 2003) de ninfas e adultos de cigarrinhas (Hemiptera, Cicadellidae) transmissoras do Mal-de-Pierce (doença causada pela bactéria Xylella fastidiosa Wells et al., 1987). 
O cultivo de citros é uma das principais atividades dos fruticultores brasileiros. A procura por frutos de melhor qualidade e com baixo teor de agrotóxicos encontra-se atualmente no topo da lista de exigências dos mercados consumidores nacionais e internacionais. A busca por metodologias alternativas de produção e o crescimento de problemas gerados por pragas transmissoras de doenças incentivam o estudo de sua ecologia e a busca de metodologias alternativas de controle integrado de pragas. Um exemplo disto é o caso da Clorose Variegada dos Citros (CVC), doença também causada pela bactéria $X$. fastidiosa, que tem como vetor principal cigarrinhas da família Cicadellidae, as quais figuram entre as presas potenciais de aranhas em agroecossistemas (Medina, 1994).

Este trabalho tem como objetivo realizar o levantamento de espécies de aranhas e fornecer dados sobre sua abundância e diversidade na vegetação herbácea e nas laranjeiras de pomares com duas diferentes formas de manejo, servindo de base para futuros estudos que visem o controle biológico de espécies de importância agrícola através deste grupo de predadores.

\section{MATERIAL E MÉTODOS}

O estudo foi realizado amostrando-se dois microhabitats (vegetação herbácea e laranjeiras) de dois pomares de Citrus sinensis (Rutaceae), variedade "Valência", distantes entre si cerca de $40 \mathrm{~km}$, com diferentes tipos de manejo: "ecológico" e "tradicional".

A composição florística da região dos Vales do Caí e Taquari é característica da Floresta Estacional Decidual; o clima apresenta dois períodos térmicos distintos: um com temperaturas médias superiores a $20^{\circ} \mathrm{C}$ (novembro a fevereiro) e outro, com temperatura média inferior a $15^{\circ} \mathrm{C}$ (junho a agosto); geralmente não são observados períodos de déficit hídrico (IBGE, 1986).

O pomar de manejo "ecológico" (ECO) localiza-se no município de Montenegro, RS $\left(51^{\circ} 33^{\prime} 36^{\prime \prime} \mathrm{W}\right.$, $29^{\circ} 31^{\prime} 27^{\prime \prime}$ 'S), onde a floresta é do tipo Submontana, relevo ondulado com solos distróficos e profundos e agrupamentos remanescentes da cobertura florestal original situados nas partes altas das encostas. As áreas menos acidentadas são ocupadas por culturas cíclicas de soja, milho, trigo, feijão e culturas perenes, como citricultura e silvicultura (IBGE, 1986).

Este pomar segue as diretrizes da Cooperativa dos Citricultores Ecológicos do Vale do Caí (Ecocitrus), que utilizam como insumos exclusivamente biofertilizantes e calda bordalesa. O pomar apresenta cerca de 200 árvores e caracteriza-se pela vegetação herbácea composta por gramíneas (Andropogon lateralis, Paspalum notatum, Brachiaria sp., Digitaria sp.), compostas (Baccharis coridifolia, B. trimera, Bidens pilosa, Vernonia sp.), leguminosas (Desmodium tortuosum, Trifolium sp., Vicea sp.), solanáceas (Solanum ciliatum e S. auriculatum) e ciperácea (Cyperus rotundus), sendo plantada batatadoce (Ipomoea batatas) como cultura intercalar.

O pomar de manejo "tradicional" (TRA) localiza-se no Centro de Pesquisa de Fruticultura de Taquari, RS (51 48'43”'W, 29 47’31'”s), órgão da Fundação Estadual de Pesquisa Agropecuária (FEPAGRO). Nesta região predomina a composição florística do tipo Floresta Montana que reveste áreas de relevo dissecado da Serra Geral, em cotas superiores a $400 \mathrm{~m}$. As condições de solo e relevo, desfavoráveis à agricultura, não impediram a devastação quase completa da cobertura florestal primitiva. Atualmente, com o progressivo abandono das áreas utilizadas na agricultura, passou a predominar a vegetação secundária em seus diversos estágios, ficando os remanescentes originais restritos às encostas íngremes e de difícil acesso (IBGE, 1986).

Este pomar apresenta cerca de 300 árvores e recebe aplicação de acaricidas, inseticidas e fungicidas. O controle da vegetação herbácea é feito entre as linhas das árvores com roçadeira e o uso periódico de herbicidas entre as laranjeiras.

Procederam dois tipos de coletas: a) na vegetação herbácea de ambos pomares e b) nas laranjeiras do pomar "ecológico". As coletas junto à vegetação herbácea de ambos os pomares foram realizadas com uma rede-devarredura de seção circular de $38 \mathrm{~cm}$ de diâmetro. As amostragens foram mensais durante fevereiro de 2001 a janeiro de $2002 \mathrm{em}$ ambos os pomares, quando foram coletadas 10 amostras a partir de pontos aleatoriamente escolhidos nas entrelinhas dos pomares; cada amostra foi composta por 50 golpes de rede em movimento de avanço (tamanho amostral por ocasião de coleta $=500$ golpes). As coletas foram efetuadas no período compreendido entre 10 e 14 horas, horário escolhido em razão do acúmulo de orvalho na vegetação durante as primeiras horas da manhã (6 a 9 horas), o que não permite um desempenho satisfatório da rede-de-varredura.

A amostragem das aranhas nas laranjeiras foi realizada exclusivamente no pomar de Montenegro (ECO), entre janeiro e dezembro de 2002. As coletas tiveram periodicidade mensal e como método de amostragem foi utilizado o guarda-chuva entomológico com área de 0,49 $\mathrm{m}^{2}$. A cada data amostral, 30 árvores foram escolhidas aleatoriamente, por sorteio, cada uma constituindo uma unidade amostral. Cada amostra foi composta por 30 batidas com um bastão de madeira nos ramos de cada uma das laranjeiras sobre um guarda-chuva entomológico. O material de cada uma das amostras foi depositado em um saco plástico de 501 , sendo refrigerado e posteriormente triado em laboratório.

Foi utilizado o teste "t pareado" para verificar a diferença entre o número total de aranhas coletadas na vegetação herbácea entre os pomares com diferentes manejos. Para tanto, foram considerados os valores de cada data amostral como um par $(\mathrm{gl}=10, \mathrm{p}=0,05)(\mathrm{ZAR}, 1999)$.

A diversidade foi determinada de acordo com os índices de Shannon-Wiener (H'), o qual expressa a diversidade pela uniformidade dos valores de importância através de todas as espécies da amostra, e Shannon (J') (Pielou), que mede a proporção da diversidade observada em relação a máxima diversidade esperada (MAGURRAN, 1988; KREBS, 1989). Para testar a significância estatística entre os índices de diversidade e o número de indivíduos foi utilizado o cálculo de Bootstrap (MoRENo, 2001).

A similaridade entre os microhabitats foi estimada de acordo com os índices de similaridade de Jaccard (presença e ausência de espécies) e Bray-Curtis (abundância das espécies) (Moreno, 2001). 
As análises de diversidade, rarefação e o teste de Bootstrap foram processados com a utilização dos programas computacionais Biodiversity Professional Beta (Bd-Pro), versão 1.0 (MCAleECE, 1997) e Paleonthological Statistical (Past), versão 1.11 (HAMMER \& HARPER, 2003).

As aranhas foram identificadas conforme bibliografia taxonômica (PlatNick, 2003). Não sendo possível a identificação específica, os exemplares foram separados em grupos constituídos por espécimes morfologicamente idênticos (morfoespécies), em nível de gênero ou família. Exemplares testemunhos estão depositados na coleção araneológica do Museu de Ciências Naturais da Fundação Zoobotânica do Rio Grande do Sul (MCN - Erica H. Buckup).

\section{RESULTADOS E DISCUSSÃO}

As amostragens totalizaram 3.876 aranhas (1.053 adultos e 2.823 jovens). Foram identificadas 17 famílias de jovens e adultos (Tab. I) e 99 espécies com base exclusivamente em adultos (Tab. II). As curvas de rarefação (Fig. 1) revelaram que as amostragens foram satisfatórias, porém sem alcançar uma tendência à estabilização horizontal (assíntota).

Considerando-se o total de aranhas adultas amostradas, as famílias Araneidae (24 spp.), Salticidae (23 spp.), Theridiidae (19 spp.), Thomisidae (10 spp.) e Anyphaenidae (8 spp.) foram as que apresentaram maior riqueza de espécies. O padrão encontrado mostra-se similar ao obtido por RiNALDI et al. (2002) em plantações de cana-de-açúcar no Brasil, onde Theridiidae, Salticidae, Anyphaenidae e Araneidae apresentaram maior número de espécies registradas. Trabalhos realizados em pomares de laranja na Austrália registraram como famílias mais ricas Araneidae, Salticidae e Thomisidae (GREen, 1996); um padrão bastante similar ao registrado neste estudo. Todavia, dados obtidos no Hemisfério Norte (AgNew et al., 1985; Dean et al., 1982; Dondale et al., 1979) indicam que Linyphiidae, Dyctinidae e Philodromidae apresentam maior número de espécies, o que difere dos resultados verificados neste estudo.

Considerando-se o total de aranhas coletadas (adultos e jovens), as errantes representaram 59,32\% nas amostras da vegetação herbácea do pomar com manejo "tradicional", 79,85\% naquelas com manejo "ecológico" e $53,17 \%$ nas amostras das laranjeiras no pomar "ecológico", dominando em ambos microhabitats estudados. Estes resultados diferem de registros do Hemisfério Norte, onde em pomares de maçã no Canadá coletas realizadas diretamente sobre as plantas revelaram que aranhas construtoras de teias foram as mais abundantes ultrapassando $70 \%$ do total coletado (Dondale et al., 1979). A maior porcentagem de aranhas errantes constatada no presente estudo pode corroborar a hipótese de JoCQué (1984), que sugere que em climas mais quentes aranhas construtoras de teias apresentem desvantagens sobre as aranhas errantes em termos de estratégias de predação.

Assembléias de aranhas na vegetação herbácea ("ecológico" x "tradicional"). Um total de 440 aranhas (115 adultas e 325 jovens) foi coletado na vegetação herbácea do pomar com manejo "tradicional" e 1.057 (177 adultas e jovens) no pomar de manejo "ecológico". Considerando-se que foi utilizado o mesmo esforço e a mesma metodologia de coleta em ambos os pomares, o número total de indivíduos coletados na vegetação herbácea foi significativamente menor no pomar "tradicional" ( $\mathrm{t}=3,482 ; \mathrm{gl}=10 ; \mathrm{p}=0,05) ;$ o baixo número de aranhas verificado na vegetação herbácea do pomar "tradicional" possivelmente está relacionado ao tipo de manejo empregado, onde a vegetação em questão é submetida ao corte raso, várias vezes ao ano.

Oxyopidae e Araneidae foram as famílias mais abundantes em ambos os pomares, somando mais de 50\% do total coletado sobre a vegetação herbácea (Tab. II). A alta dominância de Oxyopidae em ECO está possivelmente

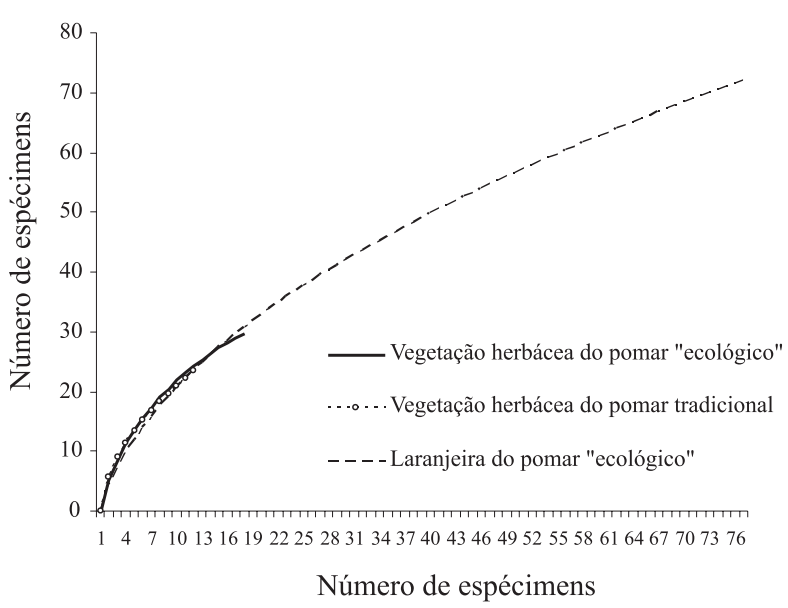

Fig. 1. Curvas de Rarefação da araneofauna coletada em vegetação herbácea e laranjeira de pomares de Citrus sinensis com manejo "ecológico" e "tradicional" nos Vales do Caí e Taquari, RS no período de fevereiro de 2001 a dezembro de 2002.

Tabela I. Composição de famílias e abundância da araneofauna (adultos e jovens) coletada na vegetação herbácea e em laranjeira de pomares de Citrus sinensis com manejo "ecológico" (ECO) e "tradicional" (TRA) nos Vales do Caí e Taquari, RS no período de fevereiro de 2001 a dezembro de 2002.

\begin{tabular}{|c|c|c|c|c|}
\hline Família & Laranjeira & $\begin{array}{c}\text { Vegetação } \\
\text { herbácea } \\
\text { ECO }\end{array}$ & $\begin{array}{c}\text { Vegetação } \\
\text { herbácea } \\
\text { TRA }\end{array}$ & Total \\
\hline Anyphaenidae & 656 & 64 & 19 & 739 \\
\hline Araneidae & 108 & 190 & 137 & 435 \\
\hline Corinnidae & 15 & 3 & 1 & 19 \\
\hline Linyphiidae & 697 & 3 & 1 & 701 \\
\hline Lycosidae & 1 & 8 & 4 & 13 \\
\hline Mimetidae & 3 & & & 3 \\
\hline Miturgidae & 64 & 17 & 35 & 116 \\
\hline Oxyopidae & 60 & 631 & 160 & 851 \\
\hline Philodromidae & 1 & & & 1 \\
\hline Pisauridae & 4 & 5 & 1 & 10 \\
\hline Salticidae & 416 & 48 & 16 & 480 \\
\hline Sparassidae & 7 & 4 & 2 & 13 \\
\hline Tetragnathidae & 7 & 12 & 40 & 59 \\
\hline Theridiidae & 295 & 8 & 1 & 304 \\
\hline Theridiosomatidae & 1 & & & 1 \\
\hline Thomisidae & 38 & 64 & 23 & 125 \\
\hline Uloboridae & 1 & & & 1 \\
\hline Indeterminado (jovem) & ) 5 & & & 5 \\
\hline Total & 2.379 & 1.057 & 440 & 3.876 \\
\hline
\end{tabular}


Tabela II. Número e porcentagem de aranhas adultas capturadas na vegetação herbácea e em laranjeiras de pomares de Citrus sinensis com manejo "ecológico" (ECO) e "tradicional" (TRA) nos Vales do Caí e Taquari, RS no período de fevereiro de 2001 a dezembro de 2002.

\begin{tabular}{|c|c|c|c|c|c|c|c|}
\hline & & & egetaçãc & espont & & Lara & jeira \\
\hline Família & Espécie & TRA & $\%$ & ECO & $\%$ & ECO & $\%$ \\
\hline Anyphaenidae & Amaurobioidinae sp. 1 & 2 & 1,73 & & & & \\
\hline & Anyphaeninae sp. 1 & & & & & 5 & 0,66 \\
\hline & Anyphaeninae sp. 2 & & & & & 2 & 0,26 \\
\hline & Arochosia honesta Keyserling, 1891 & & & & & 7 & 0,92 \\
\hline & Arochosia sp. 1 & & & & & 1 & 0,13 \\
\hline & Aysha prospera Keyserling, 1891 & & & & & 2 & 0,26 \\
\hline & Jessica osoriana (Mello-Leitão, 1922) & & & & & 4 & 0,53 \\
\hline & Jessica sp. 1 & & & & & 2 & 0,26 \\
\hline Araneidae & Acasesia aff. graciosa & 1 & 0,87 & & & & \\
\hline & Alpaida albocincta (Mello-Leitão, 1945) & & & & & 7 & 0,92 \\
\hline & Alpaida bicornuta (Taczanowski, 1878) & & & & & 1 & 0,13 \\
\hline & Alpaida hoffmani Levi, 1988 & & & & & 1 & 0,13 \\
\hline & Alpaida leucograma (White, 1841) & 9 & 7,83 & 5 & 2,82 & & \\
\hline & Alpaida veniliae (Keyserling, 1865) & 1 & & & & & \\
\hline & Araneus unanimus (Keyserling, 1879) & & & 1 & 0,56 & & \\
\hline & Argiope argentata (Fabricius, 1775) & 7 & 6,09 & 13 & 7,34 & & \\
\hline & Bertrana rufostriata (Simon, 1893) & & & & & 3 & 0,39 \\
\hline & Cyclosa machadinho (Levi, 1999) & & & & & 1 & 0,13 \\
\hline & Eustala aff. latebricola (Keyserling, 1892) & & & & & 1 & 0,13 \\
\hline & Eustala aff. taquara (Keyserling, 1892) & & & & & 1 & 0,13 \\
\hline & Eustala aff. vegeta (Keyserling, 1892) & 3 & 2,61 & 2 & 1,3 & 2 & 0,26 \\
\hline & Eustala minuscula (Keyserling, 1892) & & & 2 & 1,3 & & \\
\hline & Eustala saga (Keyserling, 1892) & 4 & 3,48 & 13 & 7,34 & 2 & 0,26 \\
\hline & Eustala sagana (Keyserling, 1892) & & & & & 1 & 0,13 \\
\hline & Gasteracantha cancriformis (Linnaeus, 1758) & & & & & 1 & 0,13 \\
\hline & Mangora sp. 1 & & & & & 1 & 0,13 \\
\hline & Ocrepeira hirsuta (Mello-Leitão, 1942) & 1 & 0,87 & 2 & 1,3 & & \\
\hline & Ocrepeira sp. 1 & 1 & 0,87 & & & & \\
\hline & Parawixia audax (Blackwall, 1863) & & & 1 & 0,56 & 3 & 0,39 \\
\hline & Scoloderus tuberculifer (O. P. Cambridge, 1889) & 1 & 0,87 & & & & \\
\hline & Verrucosa aff. arenata (Walckenaer, 1842) & & & & & 1 & 0,13 \\
\hline & Wagneriana uzaga (Levi, 1991) & & & 2 & 1,3 & & \\
\hline Corinnidae & Castianeira sp. 1 & & & & & 1 & 0,13 \\
\hline & Myrmecotypus aff. lineatipes Chickering, 1937 & & & & & 1 & 0,13 \\
\hline & Trachelopachys ignacio Platnick, 1975 & & & 1 & 0,56 & & \\
\hline Linyphiidae & Brattia sp. 1 & & & & & 1 & 0,13 \\
\hline & Erigoninae sp. 1 & & & & & 2 & 0,26 \\
\hline & Meioneta sp. 1 & & & & & 2 & 0,26 \\
\hline & Scolecura sp. 1 & & & & & 1 & 0,13 \\
\hline & Sphecozone aff. cristata Millidge, 1991 & & & & & 479 & 62,94 \\
\hline Lycosidae & Lycosidae sp. 1 & & & 2 & 1,3 & & \\
\hline Mimetidae & Mimetus hieroglyphicus Mello-Leitão, 1929 & & & & & 1 & 0,13 \\
\hline Miturgidae & Cheirachantium inclusum (Hentz, 1847) & 6 & 5,21 & 3 & 1,69 & 17 & 2,23 \\
\hline Oxyopidae & Hamataliwa constricta (Keyserling, 1891) & & & 2 & 1,3 & & \\
\hline & Oxyopes salticus Hentz, 1845 & 46 & 40,00 & 91 & 51,41 & 9 & 1,18 \\
\hline Salticidae & Aphirape uncifera (Tullgren, 1905) & & & 6 & 3,39 & 7 & 0,92 \\
\hline & Ashtabula aff. sexguttata Simon, 1901 & 1 & 0,87 & & & & \\
\hline & Beata aenea (Mello-Leitão, 1945) & & & & & 2 & 0,26 \\
\hline & Chira thysbe Simon, 1902 & & & & & 13 & 1,71 \\
\hline & Coryphasia albibarbis Simon, 1902 & & & & & 1 & 0,13 \\
\hline & Cotinusa septenpunctata Simon, 1900 & & & & & 1 & 0,13 \\
\hline & Cotinusa sp. 1 & 4 & 3,48 & 1 & 0,56 & 2 & 0,26 \\
\hline & Cotinusa sp. 2 & & & & & 1 & 0,13 \\
\hline & Cotinusa trifasciata (Mello-Leitão, 1943) & & & & & 1 & 0,13 \\
\hline & Cotinusa vittata Simon, 1900 & & & 1 & 0,56 & & \\
\hline & Dendryphantes sp. 1 & 1 & 0,87 & 1 & 0,56 & 18 & 2,37 \\
\hline & Dendryphantes sp. 2 & & & & & 5 & 0,66 \\
\hline & Dendryphantes sp. 3 & & & & & 1 & 0,13 \\
\hline & Dendryphantes sp. 4 & & & & & 1 & 0,13 \\
\hline & Frigga kessleri (Taczanowski, 1872) & & & 2 & 1,3 & 1 & 0,13 \\
\hline & Frigga quintensis (Tullgren, 1905) & & & & & 1 & 0,13 \\
\hline & Sassacus helenicus (Mello-Leitão, 1943) & & & & & 2 & 0,26 \\
\hline & Sassacus sp. 1 & 1 & & & & 0,87 & \\
\hline & Semora aff. trochilus Somin, 1901 & & & 1 & 0,56 & & \\
\hline & Tacuna delecta Peckham \& Peckham, 1901 & & & & & 2 & 0,26 \\
\hline & Thiodina aff. germaini Simon, 1900 & & & & & 6 & 0,79 \\
\hline & Unidentatae sp. 1 & 1 & 0,87 & & & & \\
\hline & Wedoquella denticulata Galiano, 1984 & & & & & 1 & 0,13 \\
\hline
\end{tabular}




\begin{tabular}{|c|c|c|c|c|c|c|c|}
\hline Sparassidae & Olios rubrosignatus (Mello-Leitão, 1943) & & & 1 & 0,56 & & \\
\hline \multirow[t]{2}{*}{ Tetragnathidae } & Leucauge volupis (Keyserling, 1893) & 17 & 14,78 & 1 & 0,56 & & \\
\hline & Tetragnatha aff. jaculator Tullgren, 1910 & 1 & 0,87 & & & & \\
\hline \multirow[t]{19}{*}{ Theridiidae } & Achaearanea aff. isana Levi, 1963 & & & & & 1 & 0,13 \\
\hline & Achaearanea altiventer (Keyserling, 1884) & & & & & 18 & 2,37 \\
\hline & Achaearanea tesselata (Keyserling, 1884) & & & & & 2 & 0,26 \\
\hline & Anelosimus ethicus (Keyserling, 1884) & & & & & 30 & 3,94 \\
\hline & Argyrodes longissimus (Keyserling, 1891) & & & & & 1 & 0,13 \\
\hline & Argyrodes rioensis Exline \& Levi, 1962 & & & & & 1 & 0,13 \\
\hline & Argyrodes sp. 1 & & & & & 1 & 0,13 \\
\hline & Argyrodes sp. 2 & & & & & 1 & 0,13 \\
\hline & Chrisso pulcherrima (Mello-Leitão, 1917) & & & 3 & 1,69 & 44 & 5,78 \\
\hline & Chrisso rubrovittata (Keyserling, 1884) & & & & & 8 & 1,05 \\
\hline & Coleosoma sp. 1 & & & 1 & 0,56 & 7 & 0,92 \\
\hline & Dipoena aff. plaumanni Levi, 1963 & & & & & 1 & 0,13 \\
\hline & Emertonella aff. emertoni (Bryant, 1933) & 1 & 0,87 & 1 & 0,56 & & \\
\hline & Emertonella taczanowski (Keyserling, 1886) & & & & & 5 & 0,66 \\
\hline & Episinus sp. 1 & & & & & 1 & 0,13 \\
\hline & Euryopis aff. spinifera (Mello-Leitão, 1944) & & & & & 1 & 0,13 \\
\hline & Theridion aff. plaumanni Levi, 1963 & & & & & 2 & 0,26 \\
\hline & Theridulla gonygaster (Simon, 1873) & 1 & 0,87 & 2 & 1,3 & 1 & 0,13 \\
\hline & Wamba aff. crispulus (Simon, 1895) & & & & & 2 & 0,26 \\
\hline \multirow[t]{9}{*}{ Thomisidae } & Misumenops argenteus (Mello-Leitão, 1929) & & & 2 & 1,3 & & \\
\hline & Misumenops pallens (Keyserling, 1880) & & & & & 1 & 0,13 \\
\hline & Misumenops pallidus (Keyserling, 1880) & 3 & 2,60 & 4 & 2,26 & 1 & 0,13 \\
\hline & Misumenops sp. 1 & 1 & 0,87 & 8 & 4,51 & & \\
\hline & Misumenops sp. 2 & & & & & 1 & 0,13 \\
\hline & Misumenops sp. 3 & & & & & 1 & 0,13 \\
\hline & Tmarus aff. pugnax Mello-Leitão, 1929 & & & & & 1 & 0,13 \\
\hline & Tmarus sp. 1 & & & 2 & 1,3 & & \\
\hline & Tmarus sp. 2 & 1 & 0,87 & & & & \\
\hline Total & & 115 & 100 & 177 & 100 & 761 & 100 \\
\hline
\end{tabular}

relacionada à vegetação mais alta e densa, à restrição do uso de herbicidas e ao número reduzido de roçadas no pomar "ecológico", o que resultou em grande densidade da vegetação herbácea no mesmo. A grande densidade da vegetação herbácea pode constituir um fator limitante para a instalação das teias espaciais de algumas espécies da família Araneidae, favorecendo ao mesmo tempo a família Oxyopidae (aranhas errantes) pelo aumento de locais para caça e abrigo.

Oxyopes salticus Hentz, 1845 foi a espécie mais abundante na vegetação herbácea de ambos os pomares (Tab. II); é bastante comum em agroecossistemas (MedinA, 1994), sendo registrada preferencialmente em ambientes de vegetação rasteira ou arbustiva (DEAN et al., 1982; Agnew et al., 1985). Destacam-se ainda: Argiope argentata (Fabricius, 1775), bem representada em ambos os pomares; Eustala saga (Keyserling, 1892), com maior representatividade no pomar "ecológico" e Leucauge volupis (Keyserling, 1893) e Alpaida leucograma (White, 1841) que apresentaram maior abundância relativa no pomar "tradicional" (Tab. II).

O número de espécies registradas para a vegetação herbácea foi maior em ECO (30 spp.) do que em TRA (24 spp.); todavia os resultados obtidos não apresentaram diferença significativa em termos de riqueza, o que é confirmado pela rarefação (Fig. 1) e pelo teste de Bootstrap para os resultados dos índices de diversidade aplicados (Tab. III). Foi registrada baixa similaridade em termos de ausência e presença de espécies entre as assembléias dos pomares estudados (índice de Jaccard $=35 \%$ ), sendo que apenas 14 espécies foram comuns aos dois pomares (Tab. II). Por outro lado, o resultado obtido para a similaridade em termos de abundância das espécies em cada assembléia amostrada foi consideravelmente maior (índice de Bray-Curtis = $52 \%)$. Percebe-se que, apesar de haver diferenças marcantes no número total de indivíduos e na composição das assembléias estudadas, a diferença na estrutura destas assembléias (considerando-se a abundância relativa das espécies) é menos evidente, o que se reflete em uma similar homogeneidade e equitabilidade.

Assembléias de aranhas no pomar "ecológico" (laranjeira x vegetação herbácea). Um total de 2.379 aranhas (761 adultas e 1.618 jovens) foi coletado nas laranjeiras do pomar "ecológico" e, como já citado anteriormente, 1.057 (177 adultas e 880 jovens) foram obtidas na vegetação herbácea do mesmo pomar "ecológico", somando 89 espécies em ambos microhabitats. Embora não tenha sido possível testar as diferenças entre o número de aranhas amostradas entre os dois tipos de configurações vegetais, em função do esforço amostral empregado (diferentes métodos de coleta) e dos períodos amostrais não-concomitantes, verificou-se que números proporcionais de adultos e jovens diferiram entre os microhabitats (laranjeiras: $65 \%$ jovens e $80 \%$ adultos, vegetação herbácea: $35 \%$ jovens e $20 \%$ adultos). Considerando-se que ambos métodos de coleta empregados permitem comparações entre as proporções amostradas, uma vez que fornecem dados de densidade absoluta de indivíduos (Southwood, 1966), a diferença na proporção de jovens e adultos pode sugerir uma preferência dos indivíduos jovens pelo ambiente das laranjeiras.

Embora não seja possível testar estatisticamente 
estas diferenças, devido a pseudoreplicação temporal, as 72 espécies registradas para as laranjeiras é consideravelmente maior do que as 30 espécies obtidas na vegetação herbácea. O índice de diversidade de Shannon-Wiener (homogeneidade) verificado nas laranjeiras foi considerado baixo (Tab. IV) o que, em razão do número total de espécies registradas, resultou em baixa eqüitabilidade. É possível que a diferença no número total de espécies coletadas em cada ambiente esteja relacionada ao esforço amostral das metodologias de coleta empregadas, sendo que, neste caso, o guardachuva entomológico possa apresentar melhor performance em termos do total de indivíduos amostrados. A similaridade em função da presença e ausência de espécies entre os microhabitats foi muito baixa (Jaccard $=17,5 \%$ ) (uma vez que registraram-se apenas 13 espécies comuns entre as duas assembléias).

A composição proporcional das assembléias em nível de família também diferiu expressivamente entre os dois microhabitats. Enquanto na vegetação herbácea dominaram as famílias Oxyopidae (59,73\%) e Araneidae $(17,95 \%)$, nas laranjeiras observou-se dominância de Linyphiidae (29,25\%), Anyphaenidae (27,53\%), Salticidae $(17,50 \%)$ e Theridiidae (12,38\%). Devemos destacar ainda a ocorrência de três exemplares ( 2 jovens e 1 adulto) da família Mimetidae; outras três famílias (Philodromidae, Theridiosomatidae e Uloboridae), representadas, cada uma, por apenas um indivíduo jovem, foram coletadas exclusivamente nas laranjeiras. Em termos gerais, a planta cítrica parece apresentar maior homogeneidade na distribuição de famílias em relação à vegetação herbácea e uma menor abundância de aranhas construtoras de teias orbiculares (Araneidae e Tetragnathidae).

$\mathrm{Na}$ assembléia de aranhas registrada nas laranjeiras, Sphecozone cristata Millidge, 1991 foi absolutamente dominante, seguida por Chrisso pulcherrima (Mello-

Tabela III. Número de espécies, indivíduos, índices de diversidade e Bootstrap da araneofauna coletada na vegetação herbácea de pomares de Citrus sinensis com manejo "ecológico" (ECO) e "tradicional" (TRA) nos Vales do Caí e Taquari, RS, no período de fevereiro de 2001 a dezembro de 2002. * Não é calculável para este tipo de dado.

\begin{tabular}{lrrrr}
\hline Valores/Microhabitat & \multicolumn{2}{c}{ Vegetação espontânea } & Bootstrap \\
\cline { 1 - 2 } \cline { 5 - 5 } \cline { 5 - 5 } Espécies & ECO & & & TRA \\
Indivíduos & 177 & & 115 & 0,359 \\
Shannon-Wiener (H') & 2,133 & & 2,245 & $*, 580$ \\
Pielou (J') & 0,627 & & 0,706 & 0,121 \\
\hline
\end{tabular}

Tabela IV. Número de espécies, indivíduos, índices de diversidade e Bootstrap da araneofauna coletada em vegetação herbácea e laranjeira em pomar de Citrus sinensis com manejo "ecológico", no Vale do Caí, RS no período de fevereiro de 2001 a dezembro de 2002. * Não é calculável para este tipo de dado.

\begin{tabular}{lcccc}
\hline Valores/Microhabitat & & Vegetação herbácea & Laranjeira & Bootstrap \\
\cline { 1 - 4 } \cline { 3 - 5 } Espécies & 30 & 72 & 0,022 \\
Indivíduos & 177 & 761 & $*$ \\
Shannon-Wiener (H') & 2,11 & 1,95 & 0,414 \\
Pielou (J') & 0,627 & 0,455 & $<0,001$ \\
\hline
\end{tabular}

Leitão, 1917) e Anelosimus ethicus (Keyserling, 1884) (Tab. II). A predominância de $S$. cristata verificada nas laranjeiras merece atenção especial já que neste tipo de ambiente as espécies da família Linyphiidae apresentam, em geral, baixa ou média abundância (Dondale et al., 1979; Rinaldi et al., 2002; RinaLdi \& RUIZ, 2002). Espécies desta família são mais comumente registradas na vegetação de cobertura do solo ou junto a este (DEAn et al., 1982; Agnew et al., 1985; Costello \& DaAne, 1998).

Chrisso pulcherrima, a segunda espécie mais abundante nas laranjeiras, já foi registrada com alta abundância em coletas com guarda-chuva entomológico em plantações de cana-de-açúcar (RINALDI et al., 2002), tal fato leva a crer que pode ser comum em ambientes agrícolas (porém, naqueles que proporcionem ambientes mais afastados do solo, como a cana-de-açúcar e a planta cítrica).

Anelosimus ethicus é, aparentemente, uma aranha de comportamento pseudo-social (AgNARSSON, 2004); embora seja comumente registrada em áreas alteradas ( $\mathrm{R}$. Ott observ. pes.), sua presença abundante em áreas agrícolas constitui informação relevante.

A baixa frequiência de $O$. salticus $(1,18 \%)$ nas laranjeiras pode ser decorrente de sua preferência pelo ambiente de vegetação herbácea ou pela disputa direta com caçadoras errantes das famílias Anyphaenidae e Salticidae, muito abundantes na planta cítrica. Em pomares de maçã no Hemisfério Norte $O$. salticus também não foi registrada (Dondale et al., 1979). Trabalhos realizados em plantações de cana-de-açúcar (RINALDi et al., 2002) e cultivos de seringueira (RINALDI \& RUIZ, 2002), confirmam a baixa abundância desta espécie em agroecossistemas com espécies vegetais de maior porte.

Os dados apresentados confirmam que a manutenção de sistemas agrícolas sob o manejo "ecológico" que procura intervir de maneira menos acentuada na dinâmica da sucessão vegetal apresenta vantagem considerável em termos de abundância, mas não em relação à diversidade de espécies de aranhas. Porém um maior número de predadores, por si só, é importante para evitar a proliferação de pragas-chave. Em acréscimo, em ambientes com maior complexidade estrutural (maior quantidade e diversidade de vegetação), a vantagem de uma espécie sobre outras - em relação às suas necessidades ecológicas - é diminuída pela competição inter-específica e pela ação predatória.

Agradecimentos. Às biólogas Cristine E. Pulz, Daniele C. Silva e Jussara B. Mezzomo (Laboratório de Entomologia da Fepagro) pelo auxílio nas coletas e triagem das aranhas. Às biólogas Erica H. Buckup e Maria A. Marques (Museu de Ciências Naturais da FZB-RS) pelo auxílio na identificação de Theridiidae, Salticidae e Araneidae. Ao Eng. Agr. Fábio J. Esswein (Emater-RS) por ceder o pomar de sua propriedade para a realização deste estudo.

\section{REFERÊNCIAS BIBLIOGRÁFICAS}

Agnarsson, I. 2004. Revision and phylogenetic analysis of American ethicus and rupununi groups of Anelosimus (Araneae, Theridiidae). Zoologica Scripta 34(4):389-413.

Agnew, C. W.; Dean, D. A. \& Smith, JR., J. W. 1985. Spiders collected form peanuts and non-agricultural habitats in the Texas west cross-timbers. The Southewestern Naturalist 30(1): $1-12$.

Altieri, M. A.; Silva, E. N. \& Nicholls, C. I. 2003. O Papel da Biodiversidade no Manejo de Pragas. Ribeirão Preto, 
Holos. 226 p.

Costello, M. J. \& DaAne, K. M. 1998. Influence of ground cover on spider populations in a table grape vineyard. Ecological Entomology 23(1):33-40.

Dean, D. A.; Sterling, W. L. \& Horner, N. V. 1982. Spiders in eastern Texas cotton fields. The Journal of Arachnology 10:251-260.

Dondale, C. D.; Parent, B. \& Pitre, D. 1979. A 6-year study of spiders (Araneae) in a Quebec apple orchard. The Canadian Entomologist 111:377-380.

Gliessman, S. R. 2000. Agroecologia. Processos ecológicos em agricultura sustentável. Porto Alegre, UFRGS. 653p.

Green, J. 1996. Spiders in biological control - An Australian perspective. Revue Suisse de Zoologie vol. hors serie: 245-253.

Hammer, O. \& Harper, D. A. T. 2003. Past. Paleonthological Statistical. v. 1.18. Disponível em: <http://folk.uio.no/ ohammer/past>. Acesso em: 23.01.2005.

Hanna, R.; Zalom, F. G. \& Roltsch, W. J. 2003. Relative impact of spiders predation and cover crop on population dynamics of Erythroneura variabilis in a raisin grape vineyard. Entomologia Experimentalis et Applicata 107:177-191.

IBGE. Instituto Brasileiro de Geografia e Estatística. 1986. Levantamento dos Recursos Naturais. Rio de Janeiro, 33, p. 541-620.

JocQué, R. 1984. Considérations concernant l'abondance relative des araignés errantes et des araignés à toile vivant au nivau du sol. Revue Arachnologique 5(4):193-204.

KreBs, C. J. 1989. Ecological methodology. New York, Harper Collins. 654p.

Magurran, A. E. 1988. Ecological diversity and its measurement. New York, Chapman and Hall. 179p.

McAleece, N. 1997. Biodiversity Professional Beta 1.0, versão 1.0. The Natural History Museum and the Scottish Association for Marine Science. Disponível em: <http:// www.nhm.ac.uk/zoology/bdpro>. Acesso em: 12.08.2004.

MedinA, A. C. 1994. Las arañas: controladores naturales de insectos fitófagos em el cultivo de arroz em norte de Santander. Revista Colombiana de Entomologia 20(3):179-186.

Moreno, C. E. 2001. Métodos para medir la biodiversidad. Zaragoza, Unesco \& SEA. 83p.
Platnick, N. I. 2003. The world spider catalog, version 3.5. American Museum of Natural History. Disponível em: <http:/ /research.amnh.org/entomology/spiders/catalog81-87/ index.html>. Acesso em: 10.07.2003.

Riechert, S. E. \& Lockley, T. 1984. Spiders as biological control agents. Annual Review of Entomology 29:299-320.

RinAldi, I. M. P. \& FoRTI, L. C. 1997. Hunting spiders of woodland fragments and agricultural habitats in the Atlantic Rain forest Region of Brazil. Studies on Neotropical Fauna and Environment 32(2):244-255.

Rinaldi, I. M. P. \& Ruiz, G. R. S. 2002. Comunidades de aranhas (Araneae) em cultivos de seringueira (Hevea brasiliensis Muell. Arg.) no Estado de São Paulo. Revista Brasileira de Zoologia 19(3):781-788.

Rinaldi, I. M. P.; Mendes, B. P. \& Cady, A. B. 2002. Distribution and importance of spiders inhabiting a Brazilian sugar cane plantation. Revista Brasileira de Zoologia 19(1):271-279.

Rypstra, A. L.; Carter, P. E.; Balfour, R. A. \& Marshall, S. D. 1999. Architectural features of agricultural habitats and their impact on the spider inhabitants. The Journal of Arachnology 27(1):371-377.

Southwood, T.R.E. 1966. Ecological Methods, with particular Reference to the Study of Insect Populations. London, Methuen. 391 p.

Sunderland, K. \& Samu, F. 2000. Effects of agricultural diversification on the abundance, distribution, and pest control potential of spiders: a review. Entomologia Experimentalis et Applicata 95:1-13.

Tarabaev, C. K. \& Sheikyn, A. A. 1990. Spiders as predators in apple-tree crows in south-eastern Kasakhstan. Acta Zoologica Fennica 190:363-366.

Turnbull, A. L. 1973. Ecology of the true spiders (Araneomorphae). Annual Review of Entomology 18:305-348.

UC Davis. 2003. University of California. Pest Management Guidelines. Disponível em: <http://www.ipm.ucdavis.edu/ PMG/r302000111.html>. Acesso em 22.08.2003.

VAngsGaARD, C. 1986. Spatial distribution and dispersal of spiders in a Danish barley field. Revue Suisse de Zoologie, vol. hors série: 671-682.

Zar, J. H. 1999. Biostatistical Analysis. New Jersey, Prentice Hall. 663p.

Recebido em outubro de 2005. Aceito em março de 2007. ISSN 0073-4721

Artigo disponível em: www.scielo.br/isz

Iheringia, Sér. Zool., Porto Alegre, 97(3):321-327, 30 de setembro de 2007 